\title{
AN OPERATIONAL MODEL FOR SPATIAL PRICE THEORY
}

\author{
John G. Greenhut and Dean H. Smith*
}

\begin{abstract}
This paper uses the basic properties of a general model of spatial pricing and distribution to establish an operational model that is free of many standard restrictive assumptions. In particular, the landscape viewed is not subject to the usual homogeneity assumptions of (1) an even distribution of identical buyers and (2) constant freight rates. Rather, heterogenous distributions and demands of varying forms are assumed. The operational form that is constructed herein would enable empirical testing and application over a broad range of industries and countries. A few case examples are included in the paper to help characterize the data that empirical studies could discover in a world of heterogeneities among buyers and landscapes.
\end{abstract}

\section{INTRODUCTION}

A war has been developing over the past two decades concerning the study of spatial pricing. On the one hand, articles have been directed toward eliminating restrictive assumptions such as linear demands and monopolistic markets (e.g., Greenhut and Greenhut 1975). On the other hand, restrictive assumptions have reappeared in empirical studies where the derivation and interpretation of complex models were so intractable that simplification proved to be necessary. ${ }^{1}$

The present paper is designed to resolve conflicting needs by establishing an operational model that could uncover situations that reject or support the classical spatial theorem (decreasing deliveries at increasing delivered prices) by modelling more realistic pricing problems. This model, we propose, can also be used to identify pricing and distribution patterns that appear to be or are in fact predatory (e.g., international dumping). Phrased more generally, the model presented in this paper identifies circumstances that give rise to particular and contrasting pricing practices.

Section II of this paper outlines the optimal control characterization of spatial price theory (OC-SPT). This section reflects prior work by M. L. Greenhut, Norman, and Hung (1987) and more recent developments by J. Greenhut, M. L. Greenhut, and Smith (1991). Since an intuitive view of the basic theory suffices

\footnotetext{
*Associate Professor at Arizona State University West and Assistant Professor at Northern Arizona University, respectively. An earlier draft of this paper was presented at the Industrial Organization workshop at Texas A\&M University. We would like to thank Mike Baye, Mary Beth Deily, and M. L. Greenhut for their helpful comments. We also would like to thank anonymous referees for substantive suggestions.
} 
for the objectives of this paper, Section II provides only a brief sketch of the optimal control approach.

Section III fulfills the fundamental objective of this paper by presenting an operational counterpart to the OC-SPT model. A specific, but broadly robust, demand function referred to in the literature as exponential demand is used here (e.g., J. Greenhut and M. L. Greenhut 1975). Besides having vital features that tie in directly to the form of our general OC-SPT model, this function is most importantly estimable with present-day, econometric software programs.

The heterogeneous landscape possibilities of OC-SPT are ascribed into the operation model by selected examples in Section IV. Alternative types of spatial heterogeneity are applied. The resulting methodology, providing for positive and negative locational demand shift parameters, establishes surprising and interesting findings. Section $\mathrm{V}$ concludes the paper with suggestions for the empirical research that our basic operational model would help generate.

\section{A SKETCH OF THE OC-SPT FRAMEWORK}

A spatial seller maximizes profits by choosing the delivery schedule (DS) that supplies optimal quantities to each buying point in an endogenously chosen spatial market area. A single seller location is assumed, and no restrictions are placed on the market space, which is to say that the market space need not be linear or circular and may in fact be irregularly shaped. Buyers may be heterogeneous in terms of basic economic demands or their concentrations at different buying points in the space. The firm is assumed to be free to price discriminate over the landscape. ${ }^{2}$

Under the standard production cost assumptions, we have $C=C(Q), C_{Q}>0$, $\mathrm{C}_{\mathrm{QQ}}>0$ along with a pointwise transportation cost $\mathrm{T}$, where $\mathrm{T}$ measures the freight cost of shipping $q(r)$ units to location $r$ and is of the form $T=T(q(r), r)$, $T_{r}>0, T_{q}>0$; it follows that freight costs increase with distance. Second partials in distance $T_{n}$ and quantities $T_{q q}$ are not signed a priori, while the second cross partial $T_{r q}$ is assumed to be positive. We simply assume negatively sloping demands and an endogenously determined market area $[0, R], r=0$ being the firm's location. These demands vary with location, depending on various consumer characteristics: $p=p(q(r), r)$. The optimal control problem involves a control sales variable $q(r)$ for each location with the state variable being the cumulative quantity sold up to a given distant location $r$. Maximized profits, $\pi(q(r), Q(R), r)$ subject to $Q(0)=0, q(r) \geq 0$ and $Q^{\prime}(r)=q(r)$, require total revenues net of transportation costs being applied against production costs. The 
classical result is obtained where

$$
p_{q} q+p-T_{q}-C_{Q}=0, \forall r
$$

It is relevant to observe that the optimal $\mathrm{q}^{*}(\mathrm{r})$ establishes a delivery schedule

$$
\frac{d q^{*}}{d r}=\frac{T_{q r}-\left(p_{q r q}+p_{r}\right)}{P_{q q q}+2 p_{q}-T_{q q}}
$$

such that under the sufficiency condition, the denominator must be negative, and, accordingly, the delivery schedule's slope is opposite in sign to that of the numerator. In turn, the firm's optimal delivered price schedule (DPS) would be characterized by $p^{*}(r)=p\left(q^{*}(r), r\right)$, with its slope given by

$$
d p^{*} / d r=p_{q} d q^{*} / d r+p_{r}
$$

It follows that demand conditions over the landscape can yield both greater quantities at higher prices as distance increases, as well as the more standard results. Given this brief background, we are now in position to set forth our operational model.

\section{OPERATIONAL MODEL: DEMAND}

As M. L. Greenhut (1956) originally proposed, the spatial distribution of consumers is essential to understanding the profit maximizing behavior of producers. By generalizing the set of pointwise demand functions, the previous section presented conclusions that cannot be reached under the typical assumptions of a constant density distribution of identical consumers. It is now manifest that when goods are shipped over an economic dimension-space, time, or quality-the effects of downward sloping demand curves may be overbalanced by diverse forces, including the differences in demand and freight costs among locations.

The question follows naturally: Can OC-SPT be converted into an operational model that can be readily used? We believe so. We begin by operationalizing consumer demands.

For purposes of empirical investigation, specification of a demand function is necessary. This is normally accomplished in microeconomic-econometric analysis by assuming demand to be either linear or constantly elastic and by stating marginal revenue as a function of elasticity. Thus, the spatial model set forth in Equa- 
tion (1) can be expressed as

$$
p\left(1-\frac{1}{n}\right)-T_{q}-C_{Q}=0
$$

where $\mathbf{n}$ is the elasticity of demand. Empirical analysis would then require estimation of elasticity. It warrants emphasis that normal procedures for estimating elasticity presuppose a specific demand form. It is commonplace to use an isoelastic function such as ${ }^{3}$

$$
\ln p=a-b \ln q
$$

or equivalently

$$
p=c q^{-\mathrm{b}},
$$

where $c=e^{a}$. Elasticity is constant for these demands and equal to $1 / b$. Another often used practice is to let demand be linear, thereby allowing elasticity to increase with price along the demand curve. ${ }^{4}$ Specifically, elasticity for the linear specification

$$
p=a-b q
$$

equals $\mathrm{p} /(\mathrm{a}-\mathrm{p})$, which increases as price rises along the demand curve.

For statistical purposes, we advocate using a far more general demand function, one that in fact contains both the linear and the isoelastic demand curves as special subsets. This demand function has been analyzed by one of the present authors in past studies and for present purposes will be defined by ${ }^{5}$

$$
p=\beta-\alpha q^{x} \text {. }
$$

We refer to this function as the exponential demand curve to highlight the importance of the exponent $x$ in identifying the shape of the curve and in determining the slope of the delivered price schedule. Note that linear demand is a subset of the exponential function when $x=1$, while the isoelastic demand function specified in (6) derives when $\beta=0, \alpha=-c$, and $x=-b$.

Delivered prices can be readily obtained from (4) by substituting the elasticity of the exponential demand, $p /[x(\beta-p)]$, and then solving for $p$. By letting $\mathrm{p}^{*}$ designate the optimal delivered prices, we obtain 


$$
p^{*}=\left[x \beta+T_{q}+C_{Q}\right] /(1+x)
$$

The DPS possesses special properties under exponential demands when space is homogeneous, as specified by $\mathrm{T}_{\mathrm{qr}}=1, \mathrm{~T}_{\mathrm{qq}}=0, \mathrm{p}_{\mathrm{qr}}=0$, and $\mathrm{p}_{\mathrm{r}}=0$. Under these restrictions, the slopes of the DS and DPS given by (2) and (3) reduce respectively to

$$
\frac{d q^{*}}{d r}=\frac{1}{-\alpha x(x+1) q^{x-1}}
$$

and

$$
\frac{d p^{*}}{d r}=\frac{1}{x+1}
$$

Different slopes and levels of delivered price schedules are generated by alternative magnitudes of $x$. The more convex the demand (e.g., $x=1 / 2$ ), the lower are delivered prices and the steeper is the slope of the DPS. When $x$ approaches zero, the slope of the schedule approaches unity, with the effect that a spatial discriminatory price policy resembles nondiscriminatory FOB pricing. Oppositely, a concave demand curve from the origin $(e . g, x=2)$ generates a flatter DPS and higher delivered prices.

It is a small step to observe that if demands vary over the space, i.e., concave at some market points and convex elsewhere, the DPS would have multiple slopes and conform generally to the empirical findings recorded by M. L. Greenhut (1981).

We observe further that different values do not affect the DPS curvature per se because they do not change the monotonicity of the pointwise demand elasticities. However, the assumption of nonconstant, nonlinear marginal costs (MC) would change the DPS to the extent that alternative market boundary limits obtain, as detailed in M. L. Greenhut, Norman, and Hung (1987, chap. 7).

A generalizing by-product of the above statements would be to note that while different levels of wealth, income distributions within and between cities, levels of competition, and the like affect curvatures of the DPS, the size of, say, the city, does not (M. L. Greenhut, Norman, and Hung 1987, chap. 1). It should be further manifest (without need for details) that different combinations of heterogeneous demands over the landscape could combine in such sequence as to generate even a linear DPS, much less nonlinear schedules. In fact, the resulting delivered prices could be uniform, FOB mill, or discriminatory against distant 
buyers or even sharply discriminatory against nearer buyers. ${ }^{6}$ The last case, which would appear to be predatory pricing in countries such as the United States where the Robinson Patman statute exists, could originate for many products from a nonpredatory design.

Three final points should be made regarding exponential demands. First, an important distinction between classes of demand curves is provided by the sign of $x$. For $x>0$ (which requires $\alpha$ and $\beta>0$ ), $\beta$ is the price intercept, and the demand curve may be concave, linear, or convex (as noted earlier). However, in each of these cases, elasticity increases more than proportionately than the rise in price along the demand curve. When $\mathrm{x}<0$ (requiring $\alpha<0$ with no restrictions placed on $\beta), \beta$ is the minimum price, and no price intercept exists. Demands are always convex from the origin for the negative exponential, and three types of changes in elasticity along the demand curve can be characterized. As price rises along a negatively exponential demand curve, elasticity may: (1) increase less than proportionately to the rise in price, (2) remain constant (the isoelastic demand obtains when $\beta=0$ and $x<0$ ), or (3) decline (M. L. Greenhut 1986). It should be noted that $x \leq-1$ results in unstable (negatively sloped) DPS because MR intersects MC from below. Thus, $x$ should be constrained to values greater than -1 to generate a stable set of delivered price schedules.

Second, the demand curves given by the exponential equation produce linear delivered price schedules throughout a spatial market controlled by one production center when spatial demands are homogeneously ordered. It was demonstrated in J. Greenhut and M. L. Greenhut (1977) that only one other demand function (essentially, where $x=0$ ) can be shown to have this characteristic, namely

$$
p=\beta-\alpha \cdot \ln q \text {, }
$$

where $\alpha>0$ and $\beta$ is generally $>0$. This demand function is extremely restrictive and produces a unit increasing delivered price schedule per extra unit distance cost for all values of $\beta$ and $\alpha^{7}$ Thus, our exponential demand provides a unique and general framework of spatial pricing, that can generate constant freight absorption rates over the designated space, besides applying to heterogeneous demand conditions with either constant or varying freight absorption rates, dependent upon market forces.

Last, the exponential demands have the vital characteristic of being estimable by existing computer programs. For example, transformation of Equation (8) to

$$
p=\beta-\alpha\left[e^{\ln q}\right]^{x}
$$




$$
\begin{aligned}
& =\beta-\alpha e^{x \cdot \ln q} \\
& =\beta-\alpha\left(e^{x}\right)^{\ln q} \\
& =\gamma+\delta \cdot p^{\ln q}
\end{aligned}
$$

where $\gamma=\beta, \delta=-\alpha$, and $\rho=e^{x}$ yields the exponential form evaluated by the BIOMED computer program. The exponential demand can also be transformed to the Gompertz curve form of $y=k a^{b}$, where $y=e^{p}, k=e^{\beta}, a=e^{-\alpha}, b=e^{x}$, and $t=\ln q$. For programs not supporting the Gompertz curve or BIOMED exponential, iterative estimation can be performed on either $p=\beta-\alpha q^{x}$, where $x$ is adjusted to minimize the regression errors, or $\ln (\beta-p)=\ln \alpha+x \cdot \ln q$, with $\beta$ serving as the iterative variable. ${ }^{8}$ The microcomputer software package RATS, among others, can be programmed to search for the optimal $\beta$ by testing for minimization of the sum of the squared errors.

In short, the exponential demand function provides a far more general and inclusive form than the special, often used linear and isoelastic equations. In addition, it is adaptable to empirical study.

\section{OPERATIONAL MODEL: HETEROGENEITY}

Since economic space is not homogeneous, particularly among different countries or regions within a country, it is vital that an operational spatial model be capable of relating to heterogeneous landscapes. For such a purpose, the basic exponential demand can be broadened by allowing the coefficients to vary over space. The following equation, for example, permits each coefficient in the exponential demand to vary in a linear fashion:

$$
p=\left(a_{\beta}+b_{\beta} r\right)-\left(a_{\alpha}+b_{\alpha} r\right) \cdot q^{a_{x}+b_{x} r}
$$

Other functional forms can also be used. The quadratic would be a prime candidate for analysis since it provides added flexibility while still containing the linear case. We use (14) herein principally for illustrative purposes.

Of utmost importance in using a model such as (14) for empirical investigation is that it be estimable. In the section above, we noted that the exponential demand could be estimated by iteration of $x$ in $p=\beta-\alpha q^{x}$ and $\beta$ in $\ln (\beta-p)=\ln$ $\alpha+x \cdot \ln q$. The same technique applies in estimating (14) by restating the equation as 


$$
p=a_{\beta}+b_{\beta} r-a_{\alpha}\left(q^{a_{x}+b_{x^{r}}}\right)-b_{\alpha}\left(r q^{\left.a_{x}+b_{x^{r}}\right)}\right.
$$

and

$$
\ln \left[\left(a_{\beta}+b_{\beta} r\right)-p\right]=\ln \left(a_{\alpha}+b_{\alpha} r\right)+a_{x}(\ln q)+b_{x}(r \cdot \ln q)
$$

Iteration of $a_{x}$ and $b_{x}$ in (15) will generate direct regression estimates of $a_{\beta}, b_{\beta}$, $a_{\alpha}$, and $b_{\alpha}$, while $a_{x}$ and $b_{x}$ can be directly estimated from (16). Several software packages can be programmed to perform the iterations, as discussed above for homogeneous exponential demands.

The optimization results for delivered prices and slopes of DS and DPS that were derived for the general case in Equations (1) through (3), and for the homogeneous exponential case in (9) through (11), can be calculated for heterogeneous exponential demands-such as (14)-by derivation of $\mathrm{p}_{\mathrm{q}}, \mathrm{p}_{\mathrm{r}}, \mathrm{p}_{\mathrm{qq}}$, and $\mathrm{p}_{\mathrm{qr}}$. While simple rules of calculus are all that is needed to calculate these variables, the reduced form equations quickly become intractable to interpret and lengthy to state. Greater benefit will be achieved by looking at specific example situations.

Consider the following simple explicit form problems. Assume a constant per unit per mile transportation rate, such that $\mathrm{T}_{\mathrm{qr}}=\mathrm{t}$ and $\mathrm{T}_{\mathrm{qq}}=0$, and then evaluate three spatial horizons using three sets of local demands. We apply below the subject demands to Equations (2) and (3) in evaluating the slopes of the DS and DPS.

The first set of demands is the linear subset of the generalized exponential form. Heterogeneity of space is provided by a downward rotation in demand around the reservation price. The demands are of the form

$$
A=\{p(r)=a-b r \cdot q\}
$$

where $\mathrm{a}$ and $\mathrm{b}$ are positive parameters, and the other variables were previously defined. In terms of Equation (14), $a_{\beta}=a, b_{\beta}=0, a_{\alpha}=0, b_{\alpha}=b, a_{x}=1$, and $b_{x}=0$.

The demands in set A rotate downward as distance from the mill increases. This states that for any given location, $\mathrm{p}_{\mathrm{r}}<0$, a standard market situation exists where population densities decrease as the seller ships over increased distances from a major metropolitan area. Given the intercept value, the elasticity of demand is constant across the set at any given price. Also, the elasticity increases in magnitude as price increases along any given demand.

Our second set of demands also takes the general form of the operational model in Equation (14) but this time allows $\beta$ to vary linearly over space, as set 
forth by

$$
B=\left\{p(r)=\left(a_{\beta}+b_{\beta} r\right)-\alpha \cdot q^{x}\right\}
$$

In terms of Equation (14), $a_{\alpha}=\alpha, b_{\alpha}=0, a_{x}=1$, and $b_{x}=0$. This set may reflect spatial markets in which preferences and/or wealth become increasingly greater over distance.

Finally, we allow both $\alpha$ and $\beta$ to change simultaneously, this time by nonlinear shifts over space. This third example set of demands is defined by

$$
C=\left\{p(r)=(a-b q) r^{\gamma}\right\}
$$

and may obtain, for example, due to decreasing population density coincidental with increasing incomes. ${ }^{9}$ For set $\mathrm{C}$, at any given price, demand becomes less elastic as distance from the mill increases.

Using these three sets of demands, the appropriate partial derivatives are substituted in Equations (2) and (3) to determine the slopes of the DS and DPS, as follows.

Set A

When the demands rotate downward, the DS slopes downward according to

$$
\frac{d q^{*}}{d r}=\frac{-t}{2 b r}-\frac{q}{r}
$$

In this case, the inward rotation extends the classical spatial model conclusion, which stipulates that the DS slopes downward according to the ratio of the transport rate and the demand curve slope. Because the demand slope varies across space, the first term on the right hand side of (20) varies with location. Moreover, the inward rotation has a second effect on the DS since the second term is the ratio of $P_{r}$ to $P_{q}$. This second term therefore reflects in value the influence of diminishing demand as distance increases. Combining the cost of distance with decreasing demands results in a DS that decreases at an increasing rate.

The slope of the DPS is found to equal

$$
\frac{d p^{*}}{d r}=\frac{t}{2}
$$


As in the classical spatial model with linear demands, the firm absorbs half the freight cost; thus, the DPS slopes upward. This term is identical to the classical spatial model because of the elasticity characteristics of set $A .^{10}$

\section{Set B}

The DS and DPS slopes derive directly from (2) and (3) as

$$
\frac{d q^{*}}{d r}=\frac{b \beta-t}{\alpha x(1+x)} \cdot q^{1-x}
$$

and

$$
\frac{d p^{*}}{d r}=\frac{x \cdot b \beta+t}{1+x}
$$

Since $\mathrm{dp}^{*} / \mathrm{dr}$ is independent of $\mathrm{r}$, the slope of the DPS is linear. The delivery schedule curvature can be identified by the second order derivative ${ }^{11}$

$$
\frac{d^{2} q^{*}}{d r^{2}}=\frac{(1-\mathrm{x}) \cdot(b \beta-t)^{2}}{[\alpha x(1+x)]^{2}} \cdot q^{1-2 x}
$$

which is positive, zero, or negative when $\mathrm{x}$ is less than, equal to, or greater than unity, except when $b_{\beta}=t$, in which case the DS slope is zero.

If $\mathrm{x}$ is a positive constant, the slopes of the DS and DPS are positive, zero, or negative according to $b_{\beta} \geq t$ and $b_{\beta} \geq-t / x$, respectively. Upward shifts in demand that outpace the additional transport cost in shipping to the next location $\left(b_{\beta}>t\right)$ result in both a positively sloped DS and DPS. Lesser increases first affect the DS slope, flattening when $b_{\beta}=t$ and becoming negative for all upward demand shifts less than $t$. Declines in the demand intercept $\left(b_{\beta}<0\right)$ result in the DS becoming more negatively sloped. The delivered price schedule, meanwhile, remains positively sloped until declines in the intercept reach substantial magnitudes, specifically $b_{\beta}<-t / x$. The special case of demand homogeneity provided by $b_{\beta}=0$ (so that $-t / x<b_{\beta}<t$ ) consequently generates a negative DS slope and a positively sloped DPS (as reflected also by Equations (10) and (11) earlier).

Finally, let us observe that the slope of the linear DPS approaches the transport rate $t$ when demands are extremely convex $(x \rightarrow$ zero), while the DPS 
slope approaches $b_{\beta}$ under extreme demand concavity $(x \rightarrow \infty)$. In between, linear demands $(x=1)$ result in a slope equally weighted by $b_{\beta}$ and $t$; in fact, the slope equals $.5 b_{\beta}+.5 t$. If demand convexity changes (e.g., $x$ gets larger as income distribution declines, or differences in preferences narrow over time), the slope of the DPS approaches $b_{\beta}$, which would represent a steeper DPS if $b_{\beta}>t$ but a flatter schedule if $b_{\beta}<t$, as would be the case in a homogeneous market. Completely contradictory results could obtain in a homogeneous market compared to a heterogeneous one in which demands shift upward over space.

We obtain further conflicting results when we allow $x<0$. While the slope condition remains identical as above for the delivery schedule $\left(b_{\beta} \geq t\right)$, it becomes opposite for the DPS $\left(b_{\beta} \leq-t / x\right)$. Now, a relatively flat DPS would obtain when large upward shifts in demand prevail over space. A DPS slope matching the transport rate would maximize profits for $b_{\beta}=(1+x-t) / x$, while uniform pricing (a zero slope) would be the outcome when $b_{\beta}=-t / x$. Assuming $t=1$ and $x=-.5$, for example, $b_{\beta}=1$ and $b_{\beta}=2$ yield unitary and zero slopes, respectively. Classical theory, if not economic intuition, would suggest that higher demands at a distance would promote greater price markups; in fact, the opposite occurs in a world of negative exponential demands and heterogeneous space.

\section{Set C}

Adding a nonlinear spatial shift to the intercept term establishes an interesting counterpart to classical spatial pricing dogma. The DS has a slope of ambiguous sign and may result in an unbounded market:

$$
\frac{d q^{*}}{d r}=\frac{-t}{2 b r^{\gamma}}+\frac{\gamma(a-2 b q)}{2 b r}
$$

If a market boundary solution obtains, then the DS may be monotonically downward sloping or may first increase and then decrease. The exact result depends on the value of $\gamma$ and on the size of the transport rate t. The DPS, given by

$$
\frac{d p^{*}}{d r}=\frac{t}{2}+\frac{\gamma a r \gamma-1}{2}
$$

is unambiguously upward sloping, but the slope will increase with distance if $\gamma>1$ or decrease with distance if $\gamma<1$. 


\section{FINAL OBSERVATIONS}

Equations (2) and (3) clearly point toward ambiguity of the DS and DPS slopes. It can be shown that the general model outlined in Section II may result in increasing or decreasing quantities at increasing or decreasing prices. Obviously, the sets of demands studied in Section IV do not describe the full set of possible variations of spatial demands.

With this in mind, the following suggestions are proposed. First, the spatial horizon may be characterized by different demand types at different locations. For instance, set A may be present at locations near the seller's mill, set B under upward shifting demands that may apply at locations slightly further from the mill but downward shifting at more distant locations, while set $\mathrm{C}$ may characterize the most remote regions of the market space. Second, other demand alternatives are available for empirical and simulation analysis. Third, alternative spatial shifts could be employed, such as a Gutenburg model adaptation, which involves nonproportional spatial shifts, $p=a+c\{\sinh (d r)\}-b q$, or its negative shift counterpart, where $c$ and $d$ are additional parameters.

Our purpose in constructing sets of demands that result in varying slopes for the DS and DPS reflects a desire to model the real-world phenomena recorded by M. L. Greenhut (1981). Clearly, companies located in Seattle may sell more in Chicago than in Duluth, and more in New York than in Wheeling. In order to fully understand empirical realities, we must first approach an understanding of the underlying forces behind the demand characteristics that yield real-world results. The current paper provides a stepping-stone toward the accomplishment of that objective.

\section{ENDNOTES}

1. See Copeland (1940-41) for the perfectly elastic demand assumption. See Ben-Akiva et al. (1985), Braid (1986), and de Palma et al. (1987) for examples of inelastic demands. See Smithies (1941) and Hamilton et al. (1989) for use of linear demands. And see M. L. Greenhut (1956), Stevens and Rydell (1966), and M. L. Greenhut (1986) for emphasis on other demand types.

2. Either the firm has complete control over the transportation system, such as a pipeline, or the phantom freight is limited by the possibility of arbitrage. We implicitly assume the former while realizing the constraint on downward sloping price schedules.

3. See, for example, Pindyck and Rubinfeld (1989). 
4. For example, see Pindyck and Rubinfeld's $(1989,118-121)$ discussion of using the isoelastic and linear functions for statistical estimation of demand.

5. This is a slight variant of the form derived in J. Greenhut and M. L. Greenhut (1977) and previously utilized in J. Greenhut and M. L. Greenhut (1975).

6. Under the exponential demand function, the slope of the delivered price schedule when $\beta$ is permitted to vary over space is

$$
\frac{d p^{*}}{d r}=\frac{x}{1+x} \cdot \frac{d \beta}{d r}+\frac{1}{x+1}
$$

A linear schedule with slope $c$ obtains if $d \beta / d r=(x c+c-1) / x$. Uniform prices would prevail when $d \beta / d r=-1 / x, F O B$ mill when $d \beta / d r=1$, discriminatory against distant buyers when $d \beta / d r>1$, and negative sloping when $d \beta / d r<-1 / x$.

7. Since $\mathrm{p}_{\mathrm{q}}=-\alpha / \mathrm{x}$ for Equation (12) demands, delivered prices are derived easily from Equation (1) as $\mathrm{p}^{*}=\alpha+\mathrm{T}_{\mathrm{q}}+\mathrm{C}_{\mathrm{Q}}$. In a homogeneous space where $\alpha$ is identical for all locations, the delivered price schedule is linear with a mill price of $\alpha+C_{Q}$ and a DPS slope with respect to $T_{q}$ equal to unity.

8. The two forms can be used together to first iterate on $\beta$ given $x$, and then to estimate $x$ given $\beta$, and so forth.

9. Set $C$ demands can be estimated in a two-step process by assuming $\gamma$ and regressing $p=a \cdot r^{\gamma}-b\left(r^{\gamma} \cdot q\right)$ to yield estimates of $a$ and $b$. In turn, the values of $a$ and $b$ can be used to estimate $\gamma$ from the regression $\ln p=\ln (a-b q)+\gamma \cdot \ln r$.

10. Since the elasticities are the same for any given price across the set of demands varying in slope, the price change reflects only the increase in transport costs and thus is the same as when a homogeneous linear demand is analyzed.

11. $\frac{d^{2} q}{d r^{2}}=\frac{\partial(d q / d r)}{\partial q} \cdot \frac{d q}{d r}$

\section{REFERENCES}

Ben-Akiva, Moshe, Andre de Palma, and J-F Thisse. "Spatial Competition with Differentiated Products." CORE Discussion Paper \#8517, June 1985.

Braid, Ralph M. "Consistent Conjectural Variations and Collusion in Spatial Competition." Columbia University, Department of Economics Discussion Paper \#333, August 1986.

Copeland, M. A. "Competing Products and Monopolistic Competition." Quarterly Journal of Economics 55 (1940-41): 1-35. 
de Palma, Andre, Jose Pedro Pontes, and J-F Thisse. "Spatial Competition Under Uniform Delivered Pricing." Regional Science and Urban Economics 17 (1987): 441-449.

Greenhut, John, and M. L. Greenhut. "Spatial Price Discrimination, Competition, and Locational Effects." Economica 42 (1975): 401-419. . "Nonlinearity of Delivered Price Schedules and Predatory Pricing." Econometrica 45 (1977): 1871-1875.

Greenhut, John, M. L. Greenhut, and Dean Smith. "An Economic Policy Derived From Spatial Pricing Theory." Texas A\&M University, Department of Economics, Working Paper \#91-13, 1991.

Greenhut, M. L. Plant Location in Theory and in Practice. Chapel Hill: University of North Carolina Press, 1956. 4th Printing, Westport: Greenwood Press, 1982.

"Spatial Pricing in the U.S.A., West Germany, and Japan." Economica 48 (1981): 79-86.

"On Demand Curves and Spatial Pricing." In Spatial Pricing and Differentiated Markets, edited by G. Norman, 65-76. London: Pion Press, 1986. Greenhut, M. L., George Norman, and C-S Hung. The Economics of Imperfect Competition: A Spatial Approach. Cambridge: Cambridge University Press, 1987.

Hamilton, J., J. Thisse, and A. Weskamp. "Spatial Discrimination: Bertrand vs. Cournot in a Model of Location Choice." Regional Science and Urban Economics 19 (1989): 87-102.

Pindyck, Robert S., and Daniel L. Rubinfeld. Microeconomics. New York: Macmillan Publishing Company, 1989.

Smithies, A. "Optimal Location in Spatial Competition." Journal of Political Economy 49 (1941): 423-439.

Stevens, B. H., and C. P. Rydell. "Spatial Demand Theory and Monopoly Price Policy." Papers, Regional Science Association 17 (1966): 195-204. 